\title{
A toolbox for robust control design: an illustrative case study
}

\author{
Laurens Jacobs ${ }^{1,2}$, Maarten Verbandt ${ }^{1,2}$, Andreas De Preter ${ }^{1,3}$, Jan Anthonis ${ }^{3}$, Jan Swevers ${ }^{1,2}$, Goele Pipeleers ${ }^{1,2}$ \\ ${ }^{1}$ MECO Research Team \\ Dept. of Mechanical Engineering, KU Leuven \\ 3001 Leuven, Belgium \\ ${ }^{2}$ DMMS lab \\ Flanders Make \\ 3001 Leuven, Belgium \\ ${ }^{3}$ Octinion bvba \\ 3001 Leuven, Belgium \\ flastname@octinion.com
}

firstname. lastname@kuleuven. be

\begin{abstract}
This paper presents LCToolbox, an open-source MATLAB software package facilitating robust linear feedback controller design. Its key feature is the automation of time-consuming pre- and postprocessing steps that are inextricable from modern control design techniques. In particular, the toolbox focuses on $\mathscr{H}_{\infty}$ loop shaping for linear time-invariant (LTI) and linear parameter-varying (LPV) systems. Since these methods require an accurate model to realize their full potential, the toolbox also supports the user to estimate LTI and LPV models using state-of-the-art system identification methods. Simulation tools are available in order to evaluate identified models and control performance. As such, this toolbox aims at supporting the user throughout the complete design process. The tailored data structures, functionalities and simulation tools are illustrated by means of a running case study considering trajectory tracking of a mobile platform with a kinematic model.
\end{abstract}

Index Terms-robust control, H-infinity loop shaping, control design software, CACSD, MATLAB

\section{INTRODUCTION}

Since the early eighties, robust feedback controller design methods have been extensively studied. In particular, the introduction of the $\mathscr{H}_{\infty}$ control paradigm gained a lot of attention and has triggered major theoretical developments in the field of robust control over the past decades. By now, the mathematical framework of these methods is rather mature and efficient solution algorithms are available. Some authors even state that "the $\mathscr{H}_{\infty}$ control problem is solved" [1]. Although promising results have been published in various application fields, e.g. [2]-[6], modern controller synthesis approaches have yet to find wide acceptance in industry. The main reason for this is twofold. On the one hand, advanced mathematical tools are required to properly apply modern controller synthesis and to critically interpret the results. On the other hand, the design procedure shifts from designing open-loop transfer functions (classical compensator design) to designing appropriate weighting functions to shape closed-loop transfer functions, still requiring insight and experience. The $\mathscr{H}_{\infty}$ controller design technique, however, offers important additional features as compared to classical open-loop shaping. Recent research has shown, for instance, that different classes of nonlinear systems, of which linear parameter-varying (LPV) control is a well-known and widely studied example, could also be dealt with in a similar manner from a control engineer's perspective [7]-[10]. That is, the mathematical formulations to obtain a numerically tractable problem are different, but the high-level design formulation remains the same.

In order to make the $\mathscr{H}_{\infty}$ controller design method more accessible to control engineers in industry by resolving the obstacles mentioned earlier, a linear control toolbox for MATLAB, 'LCToolbox', is being developed. It is a successor of the work presented in [11] and is publicly available on https://github.com/meco-group/lc_toolbox.

Although The Mathworks' MATLAB software includes plenty of routines for linear system identification, controller design, and simulation, in many cases the end user is still left with tedious pre- and postprocessing steps. Typical examples are the design of weighting functions, the construction of the generalized plant, closed-loop simulations with various model types, etc. Furthermore, the support for LPV systems is currently limited to simple parameter dependencies. Since parameter-dependent identification and control is capable of improving the control performance for nonlinear plants, software tools supporting more complex parameter dependencies [12] are appealing. The open-source 'LCToolbox' focuses on integrating and interfacing existing software packages for these purposes in a consistent way for end users. These software packages consist of the readily available MATLAB tools as well as third party software and in-house developed routines. We illustrate the design procedure within this new toolbox using a mechatronic case study: trajectory tracking of a nonholonomic mobile platform with a kinematic model.

The structure of this paper is as follows. Section II defines the illustrative example we are considering. Subsequently, the system identification module of the toolbox is illustrated in section III. With these identification results, a robust controller is designed using $\mathscr{H}_{\infty}$ loop shaping in section IV. Section $\mathrm{V}$ discusses the simulation capabilities of the toolbox by a demonstration on the case study. Some concluding remarks are finally drawn in section VI. Fig. 1 presents a 


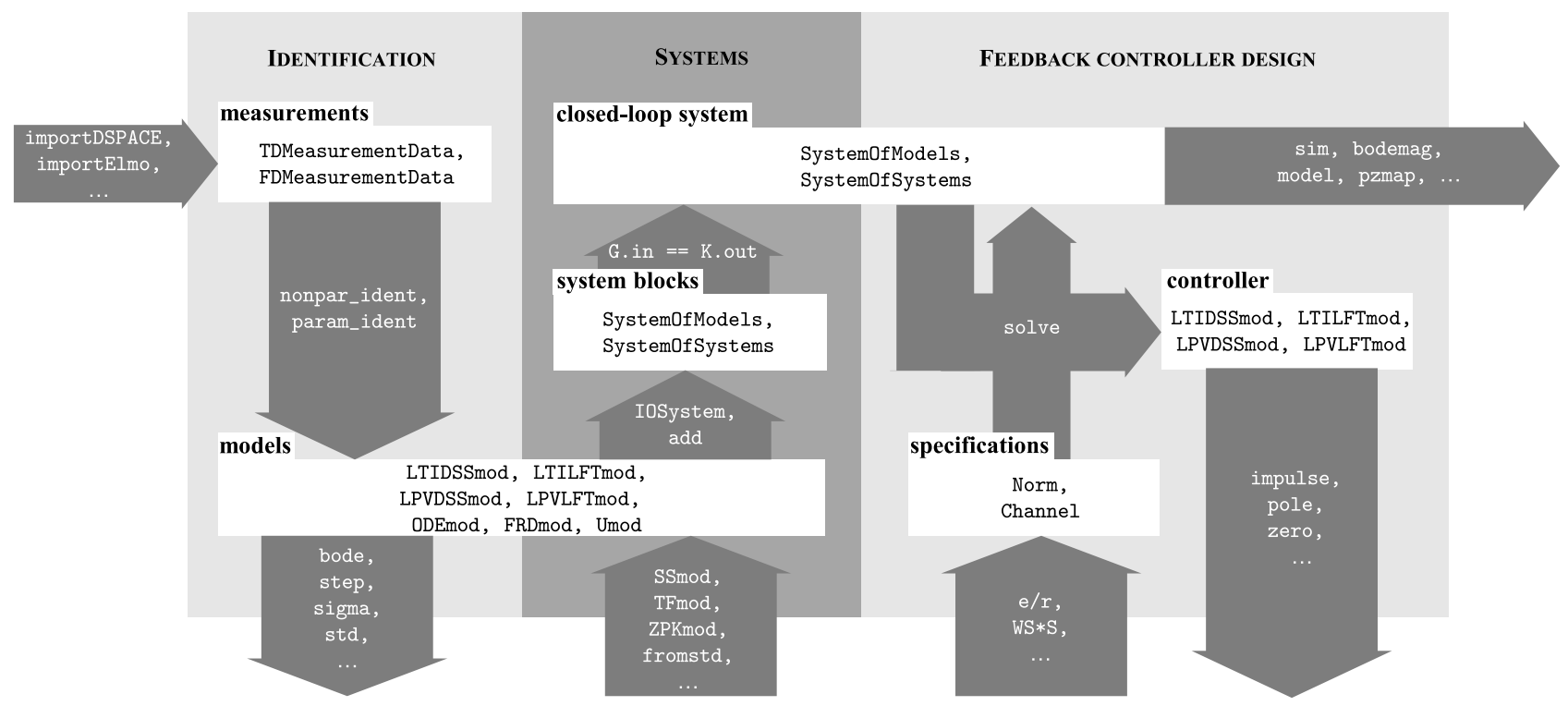

Fig. 1. Overview of the different parts of the toolbox with indication of the objects discussed in this paper. In the most general case, the user starts from measurements that are converted to LTI or LPV models by system identification methods. The resulting models are then appended to system blocks, which the user can connect to each other in order to obtain the required control configuration. By imposing specifications on this closed-loop system, the controller is calculated as the solution of the optimization problem. All measurements, systems and models can be inspected at any time once they are known.

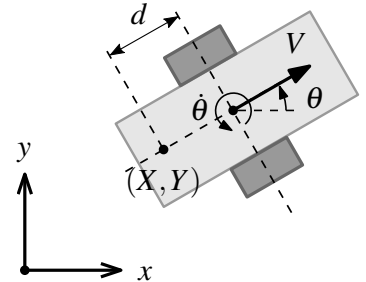

Fig. 2. Schematical representation of the Dubin's model for a simple mobile platform. The point $(X, Y)$ serves as the point to be tracked.

schematical overview of the toolbox structure, linking the different parts of the workflow that are discussed in this paper together.

\section{ILLUSTRATIVE EXAMPLE}

The nonholonomic platform of interest is a differentially driven robot, depicted in Fig. 2. The corresponding experimental setup that was used for the measurements that are reported in this paper is described in [13], apart from a slight adaptation of the wheel configuration. The motion of the center of rotation of the platform in a world frame is prescribed by (1), often referred to as the Dubin's model or the single track model. It represents the nonlinear dynamics of a system with states $(x, y, \theta)$ and control inputs $V$ and $\dot{\theta}$ :

$$
\left\{\begin{array}{l}
\dot{x}=V \cos \theta \\
\dot{y}=V \sin \theta \\
\dot{\theta}=\dot{\theta}
\end{array}\right.
$$

The relevant outputs of this system are its states. Adopting the approach of [14], the point to be tracked is chosen as $(X, Y)$, located on the center line of the platform on a nonzero distance $d$ from the center of rotation. It is readily verified in [14] that the nonlinear input transformation

$$
\left\{\begin{array}{l}
V=V_{x} \cos \theta+V_{y} \sin \theta \\
\dot{\theta}=\frac{V_{x}}{d} \sin \theta-\frac{V_{y}}{d} \cos \theta
\end{array}\right.
$$

then reduces the dynamics of $(X, Y)$ to the linear system $\dot{X}=V_{x}, \quad \dot{Y}=V_{y}$. That is, the velocity vector $\left(V_{x}, V_{y}\right)$, expressed in the global frame, becomes the new control input. Although the controllability of the orientation $\theta$ of the cart is lost, the feasibility of the reference trajectory for the nonholonomic platform naturally fixes the orientation of the platform. Since the real behavior of a differential wheeled drive deviates from (1), the feedback linearization law (2) will never exactly yield a linear system. The remaining nonlinearities are therefore considered as uncertainty on the linear model, for which the linear controller we are designing should be robustly stable and, as far as possible, guarantee robust performance. In order for the problem to remain feasible with respect to the current toolbox capabilities, we will assume that robust stability and nominal performance implies robust performance, which is not generally true for a MIMO system [15].

\section{IDENTIFICATION}

To quantify the uncertainty of the linearized model of the mobile robot, we perform identification experiments on the available setup to determine a nominal model and introduce an uncertainty model that -ideally- captures all measured deviations from the nominal model.

LCToolbox is currently being interfaced with frequency identification methods that are elaborated in detail in [16], accompagnied by their MATLAB implementations [17]. 
Linear parameter-varying identification is possible through the so-called SMILE technique [18] at present, but more advanced methods [19] are planned to be integrated as well.

\section{A. Excitation signals and measurements}

For the identification of the model of the mobile platform, two random-phase one-dimensional multisine excitation signals are generated.

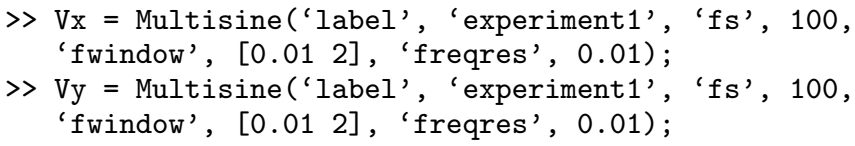

creates two (compressed) multisines, sampled at $100 \mathrm{~Hz}$, both exciting a linearly spaced frequency grid in the interval $[0.01,2] \mathrm{Hz}$. Vx and Vy are so-called TimeSignal objects, containing all information that is characteristic for these specific signals. Additional name-value pairs allow the user to specify more options, e.g. the phase distribution of the signal, its amplitude spectrum, etc. Notice that Vx and Vy are not the same, since the default phase distribution is random. TimeSignal objects feature useful methods as well. The user can for example plot their spectrum by

> plotSpectrum( $V x$, 'dft');

The actual signal content (one multisine period in this case) is obtained by

$>[\mathrm{s}, \mathrm{t}]=\operatorname{signal}(\mathrm{Vx})$;

To identify the behavior of the linearized mobile platform, a $2 \times 2$ system, at least two measurements with linearly independent excitation signals are required. This combination of two measurements (one realization) is repeated three more times, each time with different multisine excitations.

Time domain measurements are imported in the toolbox by wrapping them into TDMeasurementData objects. The constructor for such an object is called as follows:

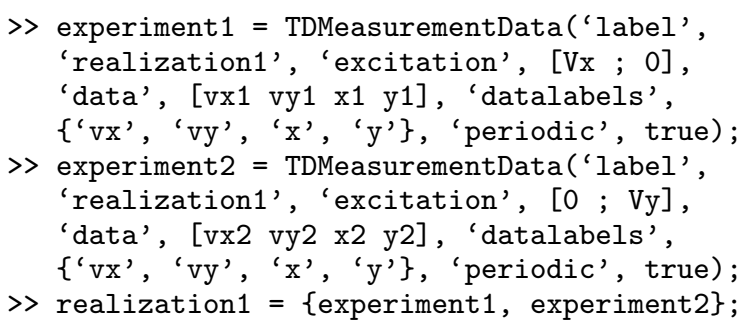

Similar to TimeSignal, this way of storing measurement data makes it possible for the control engineer to call relevant methods on the object. For example, to avoid inclusion of transient behavior in the measurement, we only keep the last 3 periods of the measurements in our case study:

> realization $1=\operatorname{clip}($ realization 1 , 'lastnper', 3);

Complementary to TDMeasurementData, an FDMeasurementData class that can handle nonparametric frequency response functions is provided as well. This class is useful in case the user prefers to perform a nonparametric identification in another software environment.

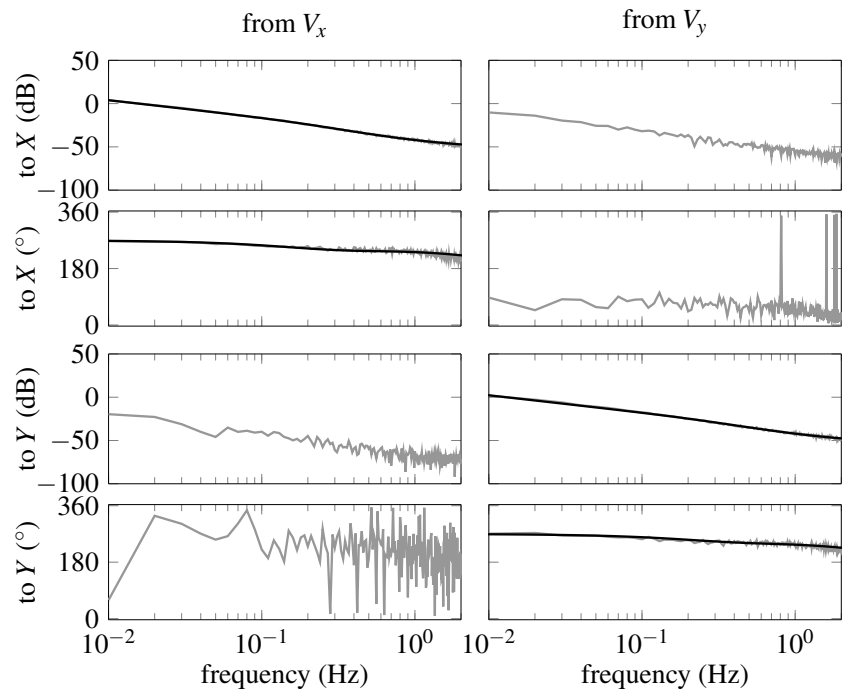

Fig. 3. Nonparametric frequency response function resulting from the identification experiments (grey) and fit of a second order linear timeinvariant (LTI) model to the diagonal components (black). The antidiagonal contributions are considered as uncertainty.

\section{B. Nonparametric frequency response function estimation}

The measured response of the system allows the user to estimate the frequency response function of the system. Essentially, the quotient of the Fourier coefficients of the measured response and the excitation signal yields the frequency response function. By comparing the coefficients obtained from experiments using different multisine realizations, the variances of the measurement noise and of the stochastic contributions of the system nonlinearities can be estimated. To this end, two methods that are elaborated in [16] are interfaced: a robust detection algorithm for nonlinearities (Robust_NL_Anal) or an algorithm based on the robust local polynomial (RLP) method (RobustLocalPolyAnal).

Returning to the mobile platform case, we calculate the average frequency response function, referred to as the best linear approximation (BLA) [16], based on the four measurements as follows:

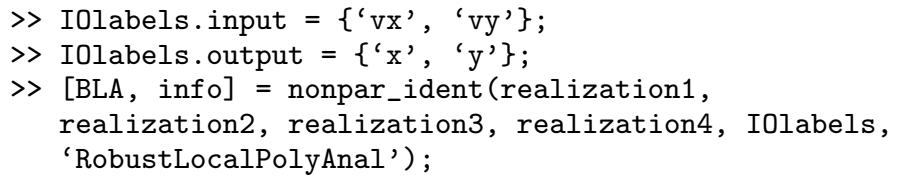

Fig. 3 shows the resulting nonparametric frequency response function, easily obtained by bode(BLA). Indeed, the linear system $\dot{X}=V_{x}, \dot{Y}=V_{y}$ is recognized for $X / V_{x}$ and $Y / V_{y}$. Nevertheless, a similar behavior is observed for the channels $X / V_{y}$ and $Y / V_{x}$, indicating the system is not completely decoupled. Since the amplitude of the antidiagonal components is considerably lower and their phase is strongly distorted, the coupling channels are considered as uncertainty on a decoupled nominal model. 


\section{Parametric frequency response function estimation}

The average nonparametric frequency response of the system is now exploited to estimate a parametric model of the linearized mobile platform. Because we consider the coupling channels as uncertainty, the MIMO fitting problem reduces to two SISO fitting problems. A second order model is proposed to capture the phase decrease observed in Fig. 3. For the time being, LCToolbox comes with the routine MIMO_ML, a maximum likelihood identification algorithm [16], MIMO_NLS, a nonlinear least-squares fitting algorithm allowing an arbitrary frequency weighting, and also interfaces MATLAB's routine tfest. The function param_ident parses the data correctly and for each of these routines:

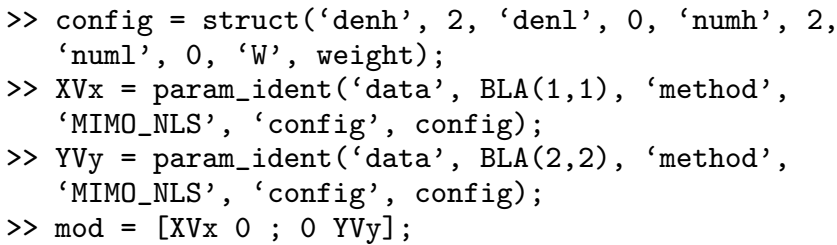

denh (numh) and denl (numl) in config represent the highest and lowest degree of the denominator (numerator) of the transfer function respectively. As shown in Fig. 3, the parametric model mod is properly fitting the diagonal channels of the BLA.

\section{ROBUST CONTROLLER DESIGN}

\section{A. Systems, models and control configurations}

The modeling layer of the toolbox makes a clear distinction between systems and models in order to facilitate the construction of the control configuration. A system (SystemOfModels or SystemOfSystems) represents a physical entity of which the true behavior between the considered input and output quantities is never exactly known. However, mathematical models of different complexities and with various representations may exist for such a system. The toolbox manages to make different models interact with each other by using its own model types. If a standard MATLAB counterpart exists, however, these are bi-directionally compatible as far as possible. Examples are SSmod (ss), TFmod (tf), ZPKmod (zpk). Nevertheless, the toolbox versions also allow these models to contain scheduling parameters, so that a standard representation is not always available. Two other relevant model types in the context of this work are ODEmod, which can handle semi-explicit differential algebraic equations of index 1 and FRDmod, the counterpart of the standard frd model, containing nonparametric frequency response data. The former, although not directly suitable for LTI or LPV control design, allows time domain simulations of closed-loop systems, which are particularly interesting for validation of the closed-loop performance of the designed controller(s). The latter is relevant to verify nonparametric frequency responses. Finally, an uncertain model class Umod (in some sense related to MATLAB's umat and uss) is

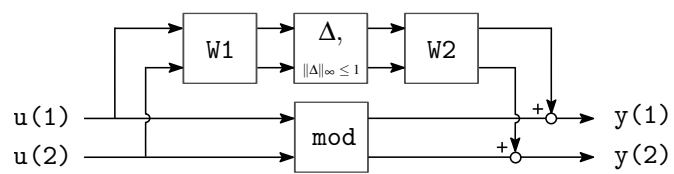

Fig. 4. An additive (unstructured) uncertainty model represents the unmodelled coupling of the parametric model.

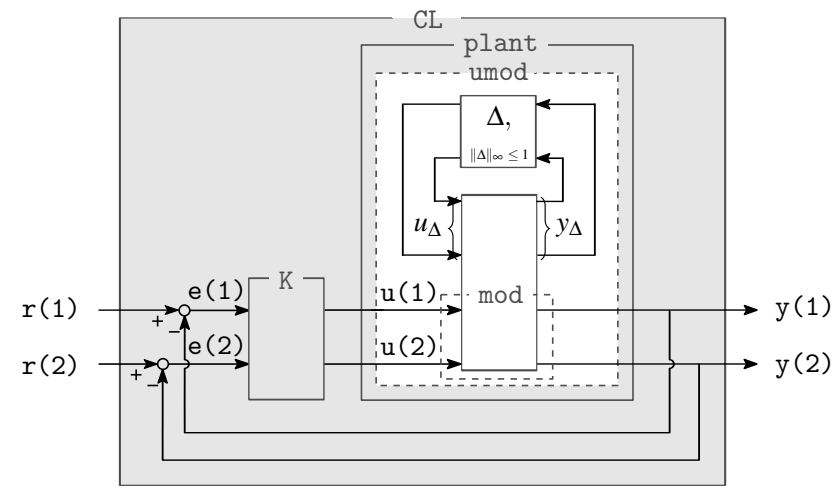

Fig. 5. Schematical representation of the control configuration that is defined in the linear control toolbox throughout this paper. Models are represented by the white boxes (mod, umod), whereas systems are represented by the grey boxes (K, plant, CL).

introduced to define an uncertain model exhibiting unstructured uncertainty, i.e. the model could be expressed as the linear fractional transformation (LFT) of a certain part and a normalized uncertain part. In the case of the mobile platform, we selected an additive uncertainty model [15] to model the deviations from the decoupled nominal identified model mod, see Fig. 4. W1 and W2 were found as appropriate weights:

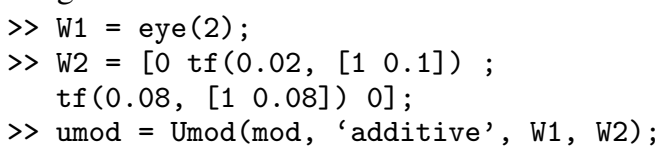

To make systems and their models interact with one another, the user has to connect the appropriate inputs and outputs by means of signals, provided by the Signal class. Signals can be assigned different names (aliases) and support convenient operations, e.g. making linear combinations. Loops are closed by stating that signals are equal to each other $(==)$. As such, the construction of the control configuration for the considered case, depicted in Fig. 5, translates to the following code:

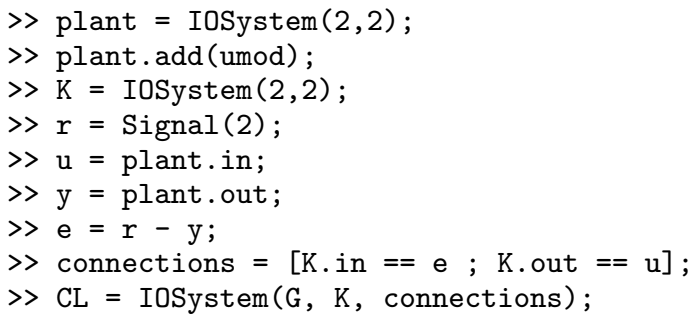

\section{B. Control problem formulation}

After having defined the control configuration, control engineers continue with the actual loop shaping. First, they have to define which input/output combinations that they 
would like to shape the closed-loop response of. These are usually referred to as channels and are represented by the Channel class. To create a sensitivity channel $\mathrm{S}$ and a complementary sensitivity channel $\mathrm{T}$, he simply invokes

$\gg \mathrm{S}=\mathrm{e} / \mathrm{r}$

$>\mathrm{T}=\mathrm{y} / \mathrm{r}$;

To enforce a certain channel shape, channels are weighted by user-defined transfer functions. Although it is possible to specify custom weights, weighting functions are typically very similar for most applications. MATLAB's makeweight generates weights with a relative degree of 0 . The toolbox additionally offers several other convenient types: weights that are used to obtain low frequency roll-off (LF), high frequency roll-off (HF) or weights to limit the peak magnitude values of the channels (DC). For the case study, second order low frequency roll-off on the sensitivity function is obtained by weighting $S$ with an LF weight WS. Furthermore, the peak value of $\mathrm{S}$ is limited to $6 \mathrm{~dB}$ by introducing a DC weight MS:

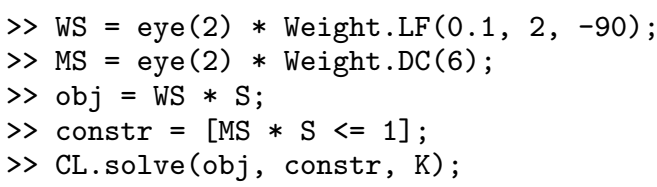

Multiplication of a weight with a channel yields (by default) an $\mathscr{H}_{\infty}$ norm (Norm). Thus, the last three lines correspond to the LCToolbox syntax to solve the $\mathscr{H}_{\infty}$ optimization problem

$$
\begin{array}{cl}
\underset{K}{\operatorname{minimize}} & \gamma \\
\text { subject to } & \left\|W_{S} S\right\|_{\infty} \leq \gamma \\
& \left\|M_{S} S\right\|_{\infty} \leq 1 \\
& \text { robust closed-loop stability }
\end{array}
$$

yielding the optimal controller $\mathrm{K}$. The attentive reader may have noticed, indeed, that the system $\mathrm{K}$ has been an 'empty box' up to now, as its content is the controller that is sought for. Therefore, the toolbox automatically appends the designed controller model to $\mathrm{K}$ after solving the controller optimization problem.

Fig. 6 shows the magnitude of the closed-loop channel $\mathrm{T}$ resulting from the aforementioned specifications. These are almost diagonal, as desired (the antidiagonal contributions are not visible due to the choice of the axes' ranges). Although the proposed weighting functions were similar, the shapes of $X / X_{\text {ref }}$ and $Y / Y_{\text {ref }}$ are not completely the same. The robust stability constraint in (3) explains this observation: due to the uncertain model umod that was used to model the plant, the toolbox automatically guarantees stability for all perturbed plants by applying the small gain theorem [15], i.e. it implicitly constrains $\left\|y_{\Delta} / u_{\Delta}\right\|_{\infty} \leq 1$ (see Fig. 5). Indeed, we can easily verify the singular values of the uncertainty channel $y_{\Delta} / u_{\Delta}$, shown in Fig. 7 . It is clear from this figure that robust stability is a constraining factor in this problem and is likely to cause the closed-loop behavior of both directions to differ.

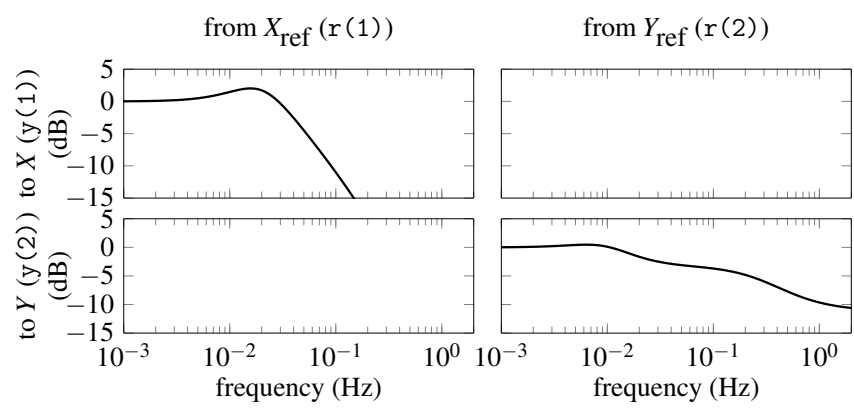

Fig. 6. Closed-loop (channel T) behavior of the synthesized controller K.

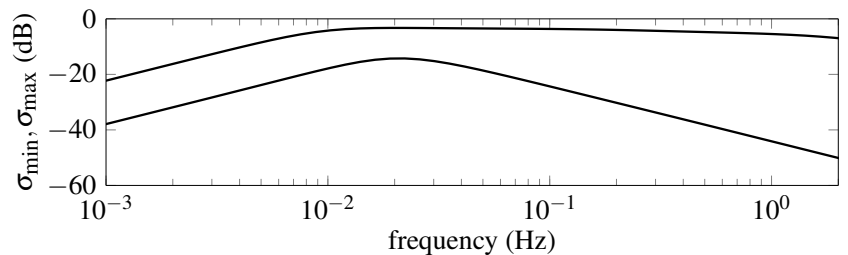

Fig. 7. Singular values of the uncertainty channel $y_{\Delta} / u_{\Delta}$ of the generalized plant for the considered control problem after controller synthesis. According to the small gain theorem, robust stability requires $\sigma_{\max } \leq 1$, which is a constraining factor in formulation (3).

\section{Simulation TOOLS}

In order to validate an identified model or to verify the performance of a feedback controller, LCToolbox offers the possibility to perform time domain simulations. Although many standard MATLAB functions are overloaded, e.g. step, impulse and lsim, the toolbox has a more generic method sim that -in addition to LTI systems- also accepts linear parameter-varying systems or even nonlinear models (ODEmod). This routine automatically assembles the appropriate set of (nonlinear) differential (algebraic) equations and feeds it to MATLAB's ODE/DAE solver ode23t behind the scenes. As a result, it becomes possible to simulate the closed-loop performance of an LTI or LPV controller for a nonlinear model.

To illustrate the simulation workflow, the performance of the controller for the trajectory tracking case is investigated. Fig. 9 shows the unit step responses of the diagonal channels of the closed-loop system (based on the identified parametric model), representing a point to point movement from $(0,0)$ to $(1,1)$. The limited bandwidth resulting from the large uncertainty causes its response to be slow. In fact, the uncertainty is too large to design a feedback controller with a high performance. Additional (nonlinear) limitations on the control signal might be present in reality, originating either from the finite bandwidth of the low level velocity control loop or from physical actuator limits. The behavior of a saturating component topping off one or multiple signals could be simply captured by an ODEmod, i.e. it could be written as

$$
\left\{\begin{array}{l}
E \dot{x}=f(x, u) \\
y=g(x, u)
\end{array}\right.
$$


with $u$ the inputs, $y$ the outputs and $x$ the states of the model. For example, if we limit the velocity commands $V_{x}$ and $V_{y}$ to the value $u 0$, one can define a system block sat as follows:

$\gg \mathrm{f}=\mathrm{Q}(\mathrm{x}, \mathrm{u})[]$;

$\gg \mathrm{g}=\mathrm{Q}(\mathrm{x}, \mathrm{u})[\max (-\mathrm{u} 0, \min (\mathrm{u}, \mathrm{u} 0))]$;

$\gg$ dimension $=[2,2,0]$

$>$ sat $=\operatorname{IOSystem}($ ODEmod $(f, g$, dimension $))$;

After building the control configuration of Fig. 8, we can calculate the step response of the diagonal channels again, while taking into account a constraint on the actuator signal:

$\gg \mathrm{u}=\mathbb{Q}(\mathrm{t})[1 ; 1]$;

$>[y, t, x]=\operatorname{sim}(\mathrm{CL}(\mathrm{r}, \mathrm{y}), \mathrm{u}, 60)$;

As could be expected, the step responses are slower and yield a larger overshoot if actuator saturation effects occur. Fig. 9 compares the step responses in the cases of unconstrained and constrained actuator signals.

\section{CONCLUSION}

This paper presented LCToolbox, a linear control toolbox for use with MATLAB. Its main purpose is to serve as an integrated toolchain for robust controller design while shielding the end user as much as possible from tedious preand postprocessing steps. It therefore has an intuitive modeling layer to represent and inspect measurements, to construct and inspect models and systems, to define control configurations, and to specify a control problem. System identification methods for linear time invariant systems and linear parameter-varying systems, controller design methods based on $\mathscr{H}_{\infty}$ optimization for LTI and LPV models, and time domain simulation tools are interfaced. The typical workflow of the software package has been demonstrated by a simple case study in progress: trajectory tracking of a Dubin's type mobile platform through feedback linearization.

\section{ACKNOWLEDGEMENT}

This research is partially supported by Flanders Make: SBO ROCSIS: Robust and Optimal Control of Systems of Interacting Subsystems. This work also benefits from KU Leuven-BOF C14/15/067: B-spline based certificates of positivity with applications in engineering, $\mathrm{KU}$ Leuven-BOF PFV/10/002: Center-of-Excellence Optimization in Engineering (OPTEC), and project FWO-G.0915.14 of the Research Foundation Flanders. Andreas De Preter is supported by a Baekeland PhD scholarship (150712) through Flanders Innovation and Entrepreneurship (VLAIO).

\section{REFERENCES}

[1] P. Apkarian and D. Noll. (2012) The $H_{\infty}$ control problem is solved https://www.math.univ-toulouse.fr/ noll/PAPERS/solved.pdf. Accessed October 20, 2017

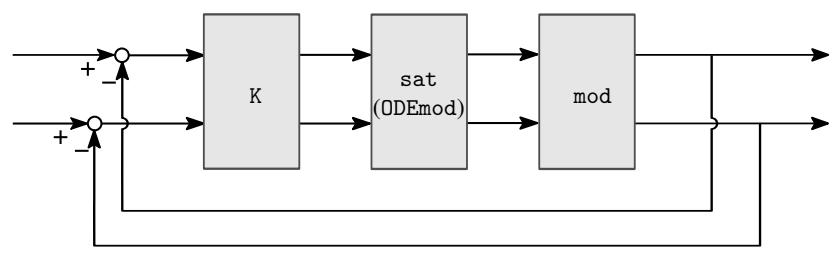

Fig. 8. Control configuration from Fig. 5, extended with a saturation block to simulate the effect of actuator saturation. Only the nominal model mod was used to verify the step responses after designing the controller. The uncertainty captured in umod is therefore not drawn here.
[2] B. Frapard and C. Champetier, " $H_{\infty}$ techniques: From research to industrial applications," in Spacecraft Guidance, Navigation and Control Systems, ser. ESA Special Publication, B. Kaldeich-Schuermann, Ed., vol. 381, Feb. 1997, p. 231.

[3] C. Lauwerys, J. Swevers, and P. Sas, "Robust linear control of an active suspension on a quarter car test-rig," Control Engineering Practice, vol. 13 , no. 5 , pp. $577-586,2005$.

[4] X. Litrico and V. Fromion, "H-infinity control of an irrigation canal pool with a mixed control politics," IEEE Transactions on Control Systems Technology, vol. 14, no. 1, pp. p. 99 - p. 111, 2006.

[5] A. M. Ousaid, S. Haliyo, S. Régnier, and V. Hayward, "H-infinity optimal control enabled micro-force sensing," in 3rd International Conference on Systems and Control, Oct 2013, pp. 490-495.

[6] C. Pittet, J. Mignot, and F. Viaud, "Attitude and active payload control: the $H_{\infty}$ revolution," in Proceedings of the 20th IFAC World Congress, July 2017, Toulouse, France.

[7] C. A. Teolis, "Robust H-infinity output feedback control for nonlinear systems," 1994.

[8] A. Isidori, " $H_{\infty}$ control via measurement feedback for affine nonlinear systems," International Journal of Robust and Nonlinear Control, vol. 4, no. 4, pp. 553-574, 1994.

[9] P. Apkarian and R. J. Adams, "Advanced gain-scheduling techniques for uncertain systems," IEEE Transactions on Control Systems Technology, vol. 6, no. 1, pp. 21-32, Jan 1998.

[10] H. C. Ferreira, P. H. Rocha, and R. M. Sales, "Nonlinear $H_{\infty}$ control and the Hamilton-Jacobi-Isaacs equation,' IFAC Proceedings Volumes, vol. 41, no. 2, pp. 188 - 193, 2008, 17th IFAC World Congress.

[11] M. Verbandt, J. Swevers, and G. Pipeleers, "An LTI control toolbox - Simplifying optimal feedback controller design," in 2016 European Control Conference (ECC), June 2016, pp. 2005-2010.

[12] G. Hilhorst, E. Lambrechts, and G. Pipeleers, "Control of linear parameter-varying systems using B-splines," in 2016 IEEE 55th Conference on Decision and Control (CDC), Dec 2016, pp. 3246-3251.

[13] R. Van Parys and G. Pipeleers, "Distributed MPC for multi-vehicle systems moving in formation," Robotics and Autonomous Systems, vol. 97, no. Supplement C, pp. 144 - 152, 2017.

[14] J. B. Pomet, B. Thuilot, G. Bastin, and G. Campion, "A hybrid strategy for the feedback stabilization of nonholonomic mobile robots," in Proceedings 1992 IEEE International Conference on Robotics and Automation, May 1992, pp. 129-134 vol.1.

[15] S. Skogestad and I. Postlethwaite, Multivariable Feedback Control: Analysis and Design. John Wiley \& Sons, 2005.

[16] R. Pintelon and J. Schoukens, System Identification: A Frequency Domain Approach. Second Edition. Wiley, 2012.

[17] _ . (2012) http://wiley.mpstechnologies.com/wiley/BOBContent/ downloadBobProjectFile.do?bpfId=1029\&bpfFileType=.zip \&bpfFileName=FreqDomBox.zip. Accessed October 20, 2017.

[18] Jan De Caigny, Juan F. Camino, and Jan Swevers, "Interpolation-based modeling of MIMO LPV systems," IEEE Trans. Contr. Sys. Techn., vol. 19 , no. 1 , pp. 46-63, 2011.

[19] D. Turk, J. Gillis, G. Pipeleers, and J. Swevers, "Identification of linear parameter-varying systems: A reweighted L2,1-norm regularization approach," Mechanical Systems and Signal Processing, vol. 100, pp. 729-742, Feb 2018

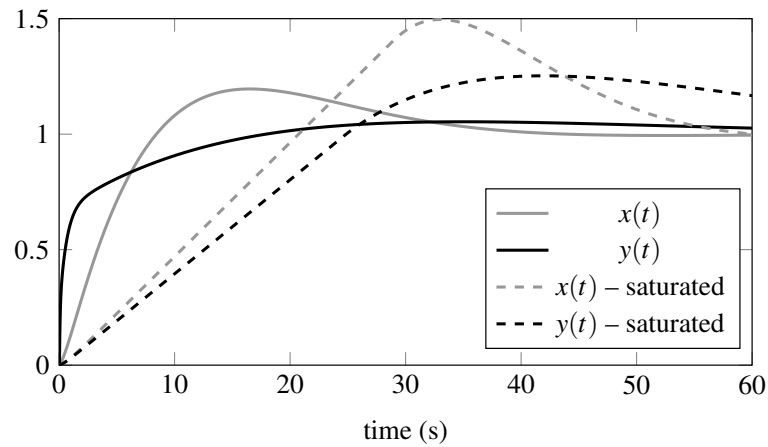

Fig. 9. Unit step responses of the diagonal channels of the closed-loop system, both without actuator saturation (calculated using the configuration of Fig. 5 and the command step) and with actuator saturation (calculated using the configuration of Fig. 8 and the command sim). 